
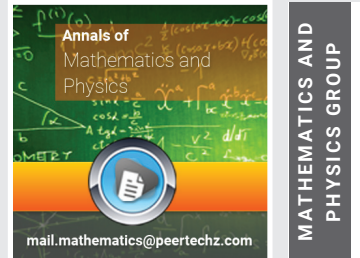

\title{
Poisson structures on (non) associative noncommutative algebras and integrable Kontsevich type Hamiltonian
}

Received: 27 December, 2019

Accepted: 29 January, 2020

Published: 30 January, 2020

*Corresponding author: Anatolij K Prykarpatski, Department of Physics, Mathematics and Computer Science, Cracov University of Technology, Krakow, Poland, E-mail: pryk.anat@cybergal.com

Keywords: Hamiltonian systems; Lie-Poisson structure; Group agebras; $\pi$-metrized Lie aleg-bras; Kontsevich type systems

https://www.peertechz.com

Check for updates systems

\section{Oksana E Hentosh ${ }^{1}$, Alexander A Balinsky² and Anatolij K Prykarpatski ${ }^{3 *}$}

${ }^{1}$ Institute for Applied Problems of Mechanics and Mathematics, NAS, Lviv, 79060 Ukraine ${ }^{2}$ Institute of Mathematics, Cardiff University, Cardiff CF24 4AG, Great Britain, UK

${ }^{3}$ Department of Physics, Mathematics and Computer Science, Cracov University of Technology, Krakow, Poland

\begin{abstract}
We have revisited the classical Poisson manifold approach, closely related to construction of Hamiltonian operators, generated by nonassociative and noncommutative algebras. In particular, we presented its natural and simple generalization allowing effectively to describe a wide class of Lax type integrable nonlinear Kontsevich type Hamiltonian systems on associative noncommutative algebras.
\end{abstract}

\section{Introduction}

We present a classical Poisson manifold approach, closely related to construction of integrable Hamiltonian systems, generated by nonassociative and noncommutative algebras. In particular, we devise its natural and simple generalization, useful for describing a wide class of Lax type integrable nonlinear Hamiltonian systems on associative noncommutative algebras, initiated first in [1-4], in case of the associative noncommutative operator algebras and continued later in [511], in case of general associative noncommutative algebras.

\section{Poisson structures on non commutative functional ma- nifolds}

It is interesting to look at construction of the Hamiltonian operators and revisit it from the classical point of view, considering them as those defined on the naturally associated [4,12-17], cotangent space $T^{*}(M)$ to some linear functional noncommutative manifold $M \simeq \widetilde{\mathbb{A}}^{*} \simeq \widetilde{\mathbb{A}}$, where $\mathbb{A}$ is, in general, a (non)associative noncommutative algebra over a field $\mathbb{K}, \widetilde{\mathbb{A}}:=C^{\infty}\left(\mathbb{S}^{1} ; \mathbb{A}\right)$ and $\widetilde{\mathbb{A}}$ is its naturally adjoint space. Then, a Hamiltonian operator on $M$ is defined [12,15], as a smooth mapping $\vartheta: M \rightarrow H o m\left(T^{*}(M) ; T(M)\right)$, such that for any fixed $u \in M$ the bracket

$$
\{f, g\}:=(\nabla f(u), \vartheta(u) \nabla g(u)),
$$

where $f, g: M \rightarrow \mathbb{K}$ are arbitrary smooth mappings from the functional space $\mathcal{D}(M) \simeq \mathcal{F}_{\widetilde{A}}(u)$, satisfies the Jacobi identity. The bracket (2.1) is determined on $M$ by means of the natural convolution $(\cdot, \cdot)$ on the product $T^{*}(M) \times T(M)$, and respectively, the gradient $\nabla f(u) \in T^{*}(M)$ of a function $f \in \mathcal{D}(M)$ is calculated as

$$
(\nabla f(u), h):=d f[u+\varepsilon h] /\left.d \varepsilon\right|_{\varepsilon=0}
$$

for any $h \in T(M)$. It is well known $[18,19]$, that a linear 
operator $\vartheta(u): T^{*}(M) \rightarrow T(M)$, determined at any point $u \in M$, is Hamiltonian iff the suitably defined [18], SchoutenNijenhuis bracket

$$
[[\vartheta(u), \vartheta(u)]]=0
$$

identically on $M$. Namely, this condition (2.3) was used in the investigations $[18,20]$, to formulate criteria for the operator $\vartheta(u): T^{*}(M) \rightarrow T(M)$ to be Hamiltonian on the functional manifold $M$. Yet these criteria appear to be very complicated and involve a large amount of cumbersome calculations even in the case of fairly simple differential expressions. So, we have reanalyzed this problem from a slightly different point of view. First, recall that the Jacobi identity for the bracket (2.1) is completely equivalent to the fact that the bracket operator defined as $D_{f}(g):=\{f, g\}$ for a fixed $f \in \mathcal{D}(M)$ and arbitrary $g \in \mathcal{D}(M)$ acts as a derivation on the space $(\mathcal{D}(M) ;\{\cdot, \cdot\})$ :

$$
D_{f}\{g, h\}=\left\{D_{f}(g), h\right\}+\left\{g, D_{f}(h)\right\},
$$

where $g, h \in \mathcal{D}(M)$ are taken arbitrary. This can be easily reformulated as follows: take any element $\varphi \in T^{*}(M)$, such that the Fréchet derivative $\varphi^{\prime}(u)=\varphi^{\prime, *}(u)$ at any $u \in M$ with respect to the convolution $(\cdot, \cdot)$ on $T^{*}(M) \times T(M)$, and construct a vector field $K: M \rightarrow T(M)$ as

$$
K(u):=\vartheta(u) \varphi(u)
$$

Then the derivation condition (2.4) can be equivalently rewritten $[4,12,15-17]$, as the strong Lie derivative

$$
L_{K} \vartheta:=\vartheta^{\prime} \cdot K-\vartheta K^{\prime, *}-K^{\prime} \vartheta=0
$$

along the vector field $K(u)=\vartheta(u) \varphi(u) \in T(M)$ at any $u \in M$ for all "self-adjoint" elements $\varphi \in T^{*}(M)$. Equivalently, a given linear skew-symmetric operator $\vartheta(u): T^{*}(M) \rightarrow T(M), u \in M$, is Hamiltonian iff the Lie derivative (2.6) vanishes for all "selfadjoint" elements $\varphi \in T^{*}(M)$. Moreover, as was observed in [21], it suffices to check the condition (2.6) only on the subspace of elements $\varphi \in T^{*}(M)$ satisfying the condition $\varphi^{\prime}(u)=0$ for any $u \in M$.

As an example, one can check that a skew-symmetric matrix-differential operator on $M$ of the form

$$
\vartheta(u):=\sigma(u) D_{x}+D_{x} \sigma^{\top}(u),
$$

where, an $n$-dimensional square matrix $\sigma(u):=\left(\sum_{s=1}^{n} u_{s} \sigma_{i j}^{s}: i, j=\overline{1, n}, n \in \mathbb{Z}_{+}\right), u \in M, \quad$ satisfies the condition (2.6) iff the linearly independent elements from $\operatorname{span}\left\{e_{j} \in \mathbb{A}: j=\overline{1, n}\right\}$ generate the finite dimensional nonassociative Balinsky-Novikov algebra [22] and satisfy the conditions $e_{i} \circ e_{j}=\sum_{s=1}^{n} \sigma_{i j}^{s} e_{s}$ for all $i, j=\overline{1, n}$. Similarly, one can verify that the skew-symmetric inverse-differential operator

$$
\vartheta(u):=\sigma(u) D_{x}^{-1}+D_{x}^{-1} \sigma(u)^{\top},
$$

where, as above $\sigma(u):=\left(\sum_{s=1}^{n} u_{s} \sigma_{i j}^{s}: u \in M, i, j=\overline{1, n}, n \in \mathbb{Z}_{+}\right)$, the sign " $T$ " means the usual matrix transposition, is Hamiltonian iff the basic nonassociative algebra $\mathbb{A}: \operatorname{span}\left\{e_{j}: j=\overline{1, n}\right\}$ coincides with the right Leibniz algebra [23] and the condition $e_{i} \circ e_{j}=\sum_{s=1}^{n} \sigma_{i j}^{s} e_{s}$ holds for any $i, j=\overline{1, n}$. The skew-symmetric inverse-differential operator (2.8) can be naturally generalized to the expression

$$
\vartheta(u):=D_{x} \sigma(u) D_{x}^{-1}-D_{x}^{-1} \sigma(u)^{\top} D_{x},
$$

which can be rewritten as

$$
\vartheta(u)=\sigma\left(D_{x} u\right) D_{x}^{-1}+D_{x}^{-1} \sigma\left(D_{x} u\right)^{\top}+\sigma(u)-\sigma(u)^{\top},
$$

where, bydefinition, $D_{x} D_{x}^{-1}=I$ and $D_{x}^{-1}(\ldots):=\frac{1}{2}\left[\int_{0}^{s} d s(\ldots)-\int_{s}^{2 \pi} d s(\ldots)\right]$ for all $x \in 0,2 \pi$ ]. The condition (2.6) for the operator (2.10) to be Hamiltonian reduces to the constraints on the related nonassociative algebra $\mathbb{A}: \operatorname{span}_{\mathbb{K}}\left\{e_{j}: j=\overline{1, n}\right\}$ exactly coinciding with those, analyzed in some detail in [24]

As it was already mentioned [18,24], based on the matrix representations of the right Leibniz algebra and the new nonassociative Riemann algebra, one can construct many nontrivial Hamiltonian operators $\vartheta(u): \mathcal{L}_{\widetilde{\mathbb{A}}} \rightarrow \mathcal{L}_{\widetilde{\mathbb{A}}}^{*}$ on the adjacent weak Lie algebra $\mathcal{L}_{\mathbb{A}}$, related with diverse types of nonassociative noncommutative algebras $\mathbb{A}$. These Hamiltonian operators prove to be very useful $[25,26,27]$, for describing a wide class of multicomponent hierarchies of integrable Riemann type hydrodynamic systems and their various physically reasonable reductions.

\section{Poisson structures on manifolds generated by associa- tive non commutative algebras}

Proceed now to a slightly generalized construction of Hamiltonian operators on a phase space, generated by associative noncommutative algebra $A$-valued matrices, which was first studied in [1-4], in case of the noncommutative operator algebras and continued later in [5-11], in case of general associative noncommutative algebras. This natural and simple generalization appeared to be very useful $[28,29,30,31,8-10]$, for describing a wide class of new Lax type integrable nonlinear Hamiltonian systems on associative noncommutative algebras, interesting for diverse applications in modern quantum physics.

We start here with a free associative noncommutative algebra $A=\mathbb{K} u_{1}, u_{2}, \ldots, u_{m}>$, generated by a finite set of elements $\left\{u_{j} \in A: j=\overline{1, m}\right\}$, and define its "abelianization" $A_{\natural}:=A /[A, A]$ and the projection $\pi: A \rightarrow A_{\natural}$, where the space $[A, A]:=\{u v-v u \in A \quad: u, v \in A\}$. Consider now a 
naturally related with $A \quad n$-dimensional matrix Lie algebra $\mathcal{G}:=\operatorname{gl}(n ; A)$ over the field $\mathbb{K}$ with entries in $A$ subject to the usual matrix commutator $[\mathrm{a}, \mathrm{b}]:=\mathrm{ab}-\mathrm{ba}$ for all $\mathrm{a}, \mathrm{b} \in \mathcal{G}$. Being first interested in the Lie-algebraic studying [14,15,32,33], of co-adjont orbits on the adjoint space $\mathcal{G}^{*}$, let us construct a bilinear form $<\cdot|\cdot\rangle: \mathcal{G} \times \mathcal{G} \rightarrow A$ on the Lie algebra $\mathcal{G}$ by means of the trace-type expression

$$
<\mathrm{a} \mid \mathrm{b}>:=\pi \operatorname{tr}\left(\mathrm{a}^{\top} \mathrm{b}\right)
$$

for any $a, b \in \mathcal{G}$. The following important lemma holds.

Lemma 3.1 The bilinear form (3.1) on $\mathcal{G}$ is symmetric, nondegenerate and ad-invariant.

Proof. Symmetricity: We have:

$$
\begin{aligned}
& <\mathrm{a} \mid \mathrm{b}>=\sum_{i, j=1, n} \pi\left(\mathrm{a}_{i j} \mathrm{~b}_{i j}\right)=\sum_{i, j=1, n} \pi\left(\mathrm{a}_{i j} \mathrm{~b}_{i j}-\mathrm{b}_{i j} \mathrm{a}_{i j}\right)+ \\
& +\sum_{i, j=1, n} \pi\left(\mathrm{b}_{i j} \mathrm{a}_{i j}\right)=\sum_{i, j=1, n} \pi\left(\mathrm{b}_{i j} \mathrm{a}_{i j}\right)=<\mathrm{b} \mid \mathrm{a}>\bmod 0_{\sharp}
\end{aligned}
$$

for any $\mathrm{a}, \mathrm{b} \in \mathcal{G}$.

Nondegeneracy: Assume that $<\mathrm{a}|\mathrm{b}\rangle=0_{\natural} \in A_{\natural}$ for a fixed $\mathrm{a} \in \mathcal{G}$ and all $\mathrm{b} \in \mathcal{G}$. state that $\mathrm{a}=0$, let us put then $\mathrm{b}=\mathrm{a}$ and obtain

$$
<\mathrm{a} \mid \mathrm{a}>=\sum_{i, j=\overline{1, n}} \pi\left(\mathrm{a}_{i j} \mathrm{a}_{i j}\right)=0_{\natural} .
$$

Taking into account that the associative algebra is generated by the finite set of elements $\left\{u_{j} \in A: j=\overline{1, m}\right\}$, it is easy to deduce from $n^{2}$ expansions of elements

$$
\begin{aligned}
& \mathrm{a}_{i j}:=c_{k(i, j)}=\sum_{\left|s^{(1)}\right| \in \mathbb{Z}_{+}} \sum_{\sigma_{1} \in \Sigma_{n}} C_{\left(k ; \sigma_{1}(1), \sigma_{1}(2), \ldots, \sigma_{1}(m)\right)}^{\left(s_{1}^{(1)} s_{2}^{(1)} \ldots s^{(1)}\right.}\left(u_{\sigma_{1}(1)}^{s_{1}^{(1)}} u_{\sigma_{1}^{2}(2)}^{s_{2}^{(1)}} \ldots u_{\sigma_{1}(m)}^{s_{m}^{(1)}}\right)+
\end{aligned}
$$

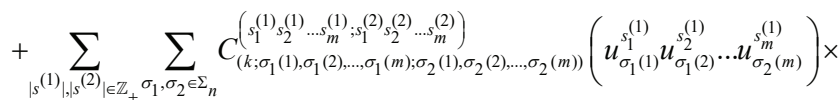

$$
\begin{aligned}
& \times\left(u_{\sigma_{1}(1)}^{s_{1}^{(2)}} u_{\sigma_{2}(2)}^{s_{2}^{(2)}} \ldots u_{\sigma_{2}(m)}^{s_{m}^{(2)}}\right)+\ldots
\end{aligned}
$$

from $A$ that the sum

$$
\sum_{k=\overline{1, n}} \pi\left(c_{k} c_{k}\right)=0
$$

iff $c_{k}=0$ for all $k=\overline{1, n^{2}}$. Really, the sum of (3.5) under the $\pi$-mapping can be now rewritten, respectively, as

$$
\tilde{\mathcal{G}}: \cup_{N \in \mathbb{Z}} \tilde{\mathrm{a}}=\sum_{j \leq N} \mathrm{a}_{j} \lambda^{j}: \mathrm{a}_{j} \in \mathcal{G}, j=\overline{1, N},
$$

$$
\begin{aligned}
& \left.\sum_{k=1, n^{2}}\left(c_{k} c_{k}\right)=\sum_{\mid s^{(1)} \in \in \mathbb{Z}_{+}} \sum_{\sigma_{1} \in \Sigma_{n}} D_{\left(\sigma_{1}(1), \sigma_{1}(2), \ldots, \sigma_{1}(m)\right)}^{\left(s_{1}^{(1)} s_{2}^{(1)} \ldots s_{m}^{(1)}\right.}\right)\left(u_{\sigma_{1}(1)}^{s_{1}^{(1)}} u_{\sigma_{1}(2)}^{s_{2}^{(1)}} \ldots u_{\sigma_{1}(m)}^{s_{m}^{(1)}}\right)+ \\
& +\sum_{\left|s^{(1)}\right|, s^{(2)} \mid \in \mathbb{Z}_{+}} \sum_{\sigma_{1}, \sigma_{2} \in \Sigma_{n}} D_{\left(\sigma_{1}(1), \sigma_{1}(2), \ldots, \sigma_{1}(m) ; \sigma_{2}(1), \sigma_{2}(2), \ldots, \sigma_{2}(m)\right)}^{\left(s_{1}^{(1)}(1) \ldots s^{(1)},(2)(2)\right.}\left(u_{\sigma_{1}(1)}^{s_{1}^{(1)}} u_{\sigma_{1}(2)}^{s_{2}^{(1)}} \ldots u_{\sigma_{2}(m)}^{s_{m}^{(1)}}\right) \times \\
& \times\left(u_{\sigma_{1}(1)}^{s_{1}^{(2)}} u_{\sigma_{2}(2)}^{s_{2}^{(2)}} \ldots u_{\sigma_{2}(m)}^{s_{m}^{(2)}}\right)+\ldots
\end{aligned}
$$

with some $D$-coefficients from $\mathbb{K}$ for all $\sigma_{j} \in S_{n}$, depending quadratically on coefficients of expansions, staying at uniform and symmetric basis elements of the algebra $A$. As the $\pi$-mapping sends all of them, by definition, to zero, the resulting system (3.5) reduces to the set of algebraic equations

$$
D_{\left(\sigma_{1}(1), \sigma_{1}(2), \ldots, \sigma_{1}(m)\right)}^{\left(s_{1}^{(1)} s_{2}^{(1)} \ldots s_{m}^{(1)}\right.}=0, D_{\left(\sigma_{1}(1), \sigma_{1}(2), \ldots, \sigma_{1}(m) ; \sigma_{2}(1), \sigma_{2}(2), \ldots, \sigma_{2}(m)\right)}^{\left(s_{1}^{(1)} s_{2}^{(1)} \ldots s_{m}^{(1)} ; s_{1}^{(2)} s_{2}^{(2)} \ldots . s_{m}^{(2)}\right)}, \ldots,
$$

reducing successively for all $\sigma_{j} \in S_{n}$ to the condintions

$$
\left.C_{\left(k ; \sigma_{1}(1), \sigma_{1}(2), \ldots, \sigma_{1}(m)\right)}^{\left(s_{1}^{(1)} s_{2}^{(1)} \ldots s_{m}^{(1)}\right)}=0, C_{\left(k ; \sigma_{1}(1), \sigma_{1}(2), \ldots, \sigma_{1}(m) ; \sigma_{2}(1), \sigma_{2}(2), \ldots, \sigma_{2}(m)\right)}^{\left(s_{1}^{(1)} s_{2}^{(1)} \ldots . . .1(1) ; s^{(2)} s_{2}^{(2)} \ldots s_{m}^{(2)}\right.}\right)
$$

being equivalent to the equalities $c_{k}=0$ for all $k=\overline{1, n^{2}}$.

As a simple consequence from Lemma 3.1 one derives the next proposition.

Proposition 3.2 The construted Lie algebra $\mathcal{G}$ is ad-invariant and $\pi$-metrized.

Proof. Really, from the symmetry property (3.2) one easily obtains that

$$
<\mathrm{a}|[\mathrm{b}, \mathrm{c}]>=<[\mathrm{a}, \mathrm{b}]| \mathrm{c}>\bmod 0_{\natural}
$$

modulo $\pi$-mapping for any elements a,b and $\mathrm{c} \in \mathcal{G}$. As the bilinear form (3.1 is non-degenerate, one has $\mathcal{G}^{*} \simeq \mathcal{G}$, that jointly with the ad-invariance property (3.9) means that the Lie algebra $\mathcal{G}$ is metrized.

Being interested in constructing integrable noncommutative dynamical systems on the algebra $A$, we need to introduce into our analysis a "spectral" parameter $\lambda \in \mathbb{C}$, responsible for the existence of infinite hierarchies of the corresponding dynamical systems invariants, guaranteeing their integrability. This wil be done in next Section, devoted to the Lie-algebraic analysis on loop-Lie-algebras, related with the Lie algebra $\mathcal{G}$, introduced above.

Consider now the Lie algebra $\{\mathcal{G},[\cdot, \cdot]\}$, constructed above, and the related loop Lie algebra $\left\{\tilde{\mathcal{G}}:=\mathcal{G} \otimes \mathbb{C}\left\{\left\{\lambda, \lambda^{-1}\right\}\right\},[\cdot, \cdot]\right\}$ of the corresponding $\mathcal{G}$-valued Laurent series with respect to the parameter $\lambda \in \mathbb{C}$,

$$
\tilde{\mathcal{G}}: \cup_{N \in \mathbb{Z}}\left\{\tilde{\mathbf{a}}=\sum_{j \leq N} \mathrm{a}_{j} \lambda^{j}: \mathrm{a}_{j} \in \mathcal{G}, j=\overline{1, N}\right\},
$$


and define on it the corresponding to (3.1) modulo $\pi$ -mapping bilinear form $(\cdot \mid \cdot): \tilde{\mathcal{G}} \times \tilde{\mathcal{G}} \rightarrow A$ :

$$
(\tilde{\mathrm{a}} \mid \tilde{\mathrm{b}}):=\operatorname{res}_{\lambda}<\tilde{\mathrm{a}} \mid \tilde{\mathrm{b}}>
$$

for any elements $\tilde{a}, \tilde{b} \in \tilde{\mathcal{G}}$. It is easy to observe that the bilinear form (3.11) is also symmetric and non-degenerate. Thus, the following proposition holds.

Proposition 3.3 The loop Lie algebra $\tilde{\mathcal{G}}$ is ad-invariant and $\pi$ -metrized.

As the loop Lie algebra $\tilde{\mathcal{G}}$ allows natural direct sum splitting $\tilde{\mathcal{G}} \quad \tilde{\mathcal{G}}_{+} \oplus \tilde{\mathcal{G}_{-}}$into two Lie subalgebras $\tilde{\mathcal{G}}_{+}$and $\tilde{\mathcal{G}}_{-}$, where

$$
\tilde{\mathcal{G}_{+}}: \cup_{N \in \mathbb{Z}_{+}}\left\{\tilde{\mathrm{a}}=\sum_{j=0, N} \mathrm{a}_{j} \lambda^{j}: \mathrm{a}_{j} \in \mathcal{G}, j=\overline{1, N}\right\}
$$

and

$$
\tilde{\mathcal{G}_{-}}: \cup_{N \in \mathbb{Z}_{+}}\left\{\tilde{\mathrm{a}}=\sum_{j \in \mathbb{Z}_{+}} \mathrm{a}_{j} \lambda^{-(j+1)}: \mathrm{a}_{j} \in \mathcal{G}, j \in \mathbb{Z}_{+}\right\},
$$

their adjoint spaces with respect to the bilinear form (3.11) split the adjoint loop space $\tilde{\mathcal{G}}^{*}=\tilde{\mathcal{G}}_{+}^{*} \oplus \tilde{\mathcal{G}}_{-}^{*}$ and satisfy the equivalences $\tilde{\mathcal{G}}_{+}^{*} \simeq \tilde{\mathcal{G}}_{-}$and $\tilde{\mathcal{G}}_{-}^{*} \simeq \tilde{\mathcal{G}}_{+}$.

Let now a linear endomorphism $\mathcal{R}: \tilde{\mathcal{G}} \rightarrow \tilde{\mathcal{G}}$ equal $\mathcal{R}=$ $\left(P_{+}-P_{-}\right) / 2$, where, by definitions, $P_{ \pm}: \tilde{\mathcal{G}} \rightarrow \tilde{\mathcal{G}} \subset \tilde{\mathcal{G}}$ are the projections on the corresponding subspaces $\tilde{\mathcal{G}_{ \pm}} \subset \tilde{\mathcal{G}}$. It is a well known property $[14,15,32,33]$ that the deformed Lie product

$$
[\tilde{\mathrm{a}}, \tilde{\mathrm{b}}]_{R}:=[\mathcal{R} \tilde{\mathrm{a}}, \tilde{\mathrm{b}}]+[\tilde{\mathrm{a}}, \mathcal{R} \tilde{\mathrm{b}}]
$$

for any $\tilde{a}, \tilde{b} \in \tilde{\mathcal{G}}$ satisfies the Jacobi condition and generates on the loop Lie algebra $\tilde{\mathcal{G}}$ a new Lie algebra structure.

Within the classical Adler-Kostant-Symes Lie-algebraic approach, or its $\mathcal{R}$-matrix structure generalization $[14,15,32,33]$, the adjoint loop space $\tilde{\mathcal{G}}^{*}$ is then endowed with the modified Lie-Poisson structure

$$
\{\tilde{1}(\tilde{a}), \tilde{1}(\tilde{b})\}_{\natural}:=\left(\tilde{1} \mid[\tilde{a}, \tilde{b}]_{\mathcal{R}}\right),
$$

for any basic functionals $\tilde{1}(\tilde{a}), \tilde{1}(\tilde{b}) \in D\left(\tilde{\mathcal{G}}^{*}\right)$ subject to which the whole set

$$
I\left(\tilde{\mathcal{G}}^{*}\right)=\left\{\gamma \in D\left(\tilde{\mathcal{G}}^{*}\right):(\tilde{1} \mid[\operatorname{grad} \gamma(\tilde{\mathrm{l}}), \tilde{a}])=0_{\natural}, \tilde{a} \in \tilde{\mathcal{G}}^{*}\right\}
$$

of smooth Casimir functionals on $\tilde{\mathcal{G}}^{*}$ is commutative with respect to the deformed Lie-Poisson structure (3.15) on $\tilde{\mathcal{G}}^{*}$, that is $\{\gamma, \mu\}_{\natural}=0_{\natural} \in A_{\natural}$ for all $\gamma, \mu \in I\left(\tilde{\mathcal{G}}^{*}\right)$ and, by definition, $(\tilde{\mathrm{q}} \mid \operatorname{grad} \gamma(\tilde{\mathrm{l}})):=\left.\frac{d}{d \varepsilon} \gamma(\tilde{\mathrm{l}}+\varepsilon \tilde{\mathrm{q}})\right|_{\varepsilon=0}$. The latter makes it possible to construct integrable Hamiltonian flows on the associative algebra $A$ as Poissonian flows on the co-adjoint orbits on the adjoint space $\tilde{\mathcal{G}}^{*}$, generated by suitable loop Lie algebra $\tilde{\mathcal{G}}$ Casimir gradient elements. Namely, if an element $\tilde{\imath} \in \tilde{\mathcal{G}}^{*}$ is fixed, the corresponding Hamiltonian flow on $\tilde{\mathcal{G}}^{*}$ subject to the deformed Poisson bracket (3.15) and a Casimir funcrtional $\gamma \in I\left(\tilde{\mathcal{G}}^{*}\right)$ possesses the well known Lax type [33-40], representation

$$
d \tilde{\mathrm{l}} / d t=\left[P_{+} \operatorname{grad} \gamma(\tilde{\mathrm{l}}), \tilde{\mathrm{l}}\right]\left(\bmod 0_{\natural}\right),
$$

where $t \in \mathbb{K}$ is a related evolution parameter. The example of this construction and its Lie algebraic properties are discussed in the next Subsection.

\section{Kontsevich type integrable systems on unital finitely ge- nerated free associative noncommutative algebras}

Let a free unital finitely generated associative non-commutative algebra $A:=\mathbb{K}<u^{ \pm}, v^{ \pm}>$be the corresponding group algebra of a group $G\{u, v\}$, generated by two elements $u, v \in G$. The algebra $A$ is infinite dimensional with the countable basis $L_{A}<1, u^{j} v^{s_{1}-j}, v^{j} u^{s_{1}-j}, u^{j} v^{s_{2}-j} u^{j-k} v^{k-q}, v^{j} u^{s_{2}-j} v^{j-k} u^{k-q}, \ldots: s_{1}, s_{2}, \ldots \in \mathbb{Z}>$, the related two-dimensional matrix loop Lie algebra $\tilde{\mathcal{G}} \mathcal{G} \otimes \mathbb{C}\left\{\left\{\lambda, \lambda^{-1}\right\}\right\}, \mathcal{G}:=\operatorname{gl}(2 ; A)$, is metrized subject to the bi-li near product (3.11) and generated by affine elements

$$
\tilde{\mathrm{a}}=\sum_{j=0,3} \sigma_{k} \sum_{j \ll \infty} \mathrm{a}_{j}^{(k)} \lambda^{j}
$$

with four basis Pauli matrix elements $\sigma_{k} \in \operatorname{gl}(2 ; \mathbb{K}), k=\overline{0,3}$, and algebra components $\mathrm{a}_{j}^{(k)} \in A, j \ll \infty, k=\overline{0,3}$. The corresponding Casimir functionals $\gamma \in I\left(\tilde{\mathcal{G}}^{*}\right)$ generate a Hamiltonian flow on points $\tilde{1} \in \tilde{\mathcal{G}}^{*}$ with respect to the Poisson bracket (3.15) in the Lax type form (3.17). To analyze this flow in detail, let us put, by definition, that the seed orbit point $\tilde{1} \epsilon$ $\tilde{\mathcal{G}}^{*}$ is given by the following $\lambda$-squared expression

$$
\tilde{1}=\sum_{j=\overline{0,3}} \sum_{k=\overline{0,2}} \sigma^{j} \lambda^{k-3} u_{j}^{(k)}
$$

where $\left\{\sigma^{j} \in g l^{*}(2 ; \mathbb{K}): \operatorname{tr}\left(\sigma^{j} \sigma_{k}\right)=\delta_{k}^{j}, j, k=\overline{0,3}\right\}$ is the dual basis of the matrix space $g l^{*}(2 ; \mathbb{K}) \simeq g l(2 ; \mathbb{K})$ and elements $\left\{u_{j}^{(k)} \in A: j=\overline{0,3}, k=\overline{0,2}\right\}$ are coordinates of some $A$-algebra valued phase space $M_{A}^{(0 \mid 2)}$ in a general position. In particular, we will choose the following dual bases:

$$
\sigma_{0}=\left(\begin{array}{ll}
1 & 0 \\
0 & 1
\end{array}\right), \sigma_{1}=\left(\begin{array}{ll}
0 & 1 \\
0 & 0
\end{array}\right), \sigma_{2}=\left(\begin{array}{ll}
0 & 0 \\
1 & 0
\end{array}\right), \sigma_{3}=\left(\begin{array}{cc}
1 & 0 \\
0 & -1
\end{array}\right)
$$

in $g l(2 ; \mathbb{K})$ and

$$
\sigma^{0}=\left(\begin{array}{cc}
1 / 2 & 0 \\
0 & 1 / 2
\end{array}\right), \sigma^{1}=\left(\begin{array}{ll}
0 & 1 \\
0 & 0
\end{array}\right), \sigma^{2}=\left(\begin{array}{ll}
0 & 0 \\
1 & 0
\end{array}\right), \sigma^{3}=\left(\begin{array}{cc}
1 / 2 & 0 \\
0 & -1 / 2
\end{array}\right)
$$

in $g l(2 ; \mathbb{K})^{*}$. Moreover, we also will assume that $A$-algebra valued coefficients of the phase space $M_{A}^{(0 \mid 2)}$ in (4.2) are representable subject to the basis of $A$ as 


$$
\begin{array}{ccccc}
\lambda \backslash \sigma & \sigma^{0} & \sigma^{1} & \sigma^{2} & \sigma^{3} \\
\lambda^{-3} & u_{0}^{(0)}=1 & u_{1}^{(0)}=u & u_{2}^{(0)}=0 & u_{3}^{(0)}=-1 \\
& u_{0}^{(1)}=v+v^{-1}+ & u_{1}^{(1)}=v^{-1} & u_{2}^{(1)}=v^{-1} u^{-1}+ & u_{3}^{(1)}=-v+v^{-1}+, \\
\lambda^{-2} & +u+u^{-1}+v^{-1} u^{-1} & & +u^{-1}+1 & +u-u^{-1}-v^{-1} u^{-1} \\
\lambda^{-1} & u_{0}^{(2)}=0 & u_{1}^{(2)}=0 & u_{2}^{(2)}=v & u_{3}^{(2)}=0
\end{array}
$$

following the result obtained in [31].

As a first important task, we will calculate the corresponding Poisson structure on the related A-algebra valued phase space $M_{A}^{(0 \mid 2)}(\tilde{1})$, generated by coefficients, presented in the expression (4.4). To do this, we need to take into account that the phase space $M_{A}^{(0 \mid 2)}(\tilde{1})$, being endowed with the $\mathcal{R}$-modified Poisson structure (3.15), is strongly reduced via the Dirac scheme [4,32], subject to the set

$$
\begin{aligned}
& \Phi:=\left\{\varphi_{1}=u_{0}^{(0)}-1=0, \varphi_{2}=u_{0}^{(2)}=0,\right. \\
& \left.\varphi_{3}=u_{2}^{(0)}=0, \varphi_{4}=u_{3}^{(2)}=0, \varphi_{5}=u_{3}^{(0)}+1=0\right\}
\end{aligned}
$$

of algebraic constraints, imposed on the phase space $M_{A}^{(0 \mid 2)}$. The latter means that the true Poisson structure on the reduced phase space $M_{A}^{(0 \mid 2)}(\tilde{1}):=M_{A}^{(0 \mid 2)} / \Phi$ coincides with the corresponding Dirac type reduction of the $\mathcal{R}$-modified Poisson structure, defined on the full phase space $M_{A}^{(0 \mid 2)}$. As a result of simple enough yet cumbersome calculations we arrive at the following Poisson brackets

$$
\{u, v\}_{\natural}=-u v,\{u, u\}_{\natural}=0_{\natural}=\{v, v\}_{\natural}
$$

on the reduced phase space $M_{A}^{(0 \mid 2)}(\tilde{1}) \simeq A:=\mathbb{K}\left\langle u^{ \pm}, v^{ \pm}\right\rangle$.

Having taken as a Hamiltonian operator $h:=\operatorname{res} \lambda^{2} \operatorname{tr}\left(\tilde{1}^{2}\right) \in I\left(\tilde{\mathcal{G}}^{*}\right)$, one easily obtains the following [5], nonlinear integrable Kontsevich dynamical system

$$
\left.\begin{array}{c}
d u / d t:=\{h, u\}_{\natural}=u v-u v^{-1}-v^{-1} \\
d v / d t:=\{h, v\}_{\natural}=-v u+v u^{-1}+u^{-1}
\end{array}\right\}:=K(u, v)
$$

on the reduced phase space $A=\mathbb{K}\left\langle u^{ \pm}, v^{ \pm}\right\rangle$. Moreover, owing to the Lax type representation (3.15), the Kontsevich dynamical system (4.7) proves to be equivalent to the following matrix commutator equation

$$
d \tilde{1} / d t=[\tilde{1}, p(\tilde{1})]\left(\bmod 0_{\natural}\right)
$$

for any $\lambda \in \mathbb{K}$ in the Lie algebra $\tilde{\mathcal{G}}$, where the $A$-valued matrix

$$
\begin{aligned}
& p(\tilde{1})=P_{+} \operatorname{gradh}(\tilde{1}) / 2 \\
= & \sigma_{0}\left(v^{-1}-v+u+1\right) / 2+\sigma_{1} \lambda v+\sigma_{2} v^{-1}+\sigma_{3}\left(v^{-1}-v+u-1\right) / 2 \in \tilde{\mathcal{G}} .
\end{aligned}
$$

Taking as Hamiltonian functions the algebraic expressions

$$
h^{(m, n)}:=\operatorname{res} \lambda^{m} \operatorname{tr}\left(\tilde{1}^{n}\right) \in I\left(\tilde{\mathcal{G}}^{*}\right), m, n \in \mathbb{Z} \text {, one can obtain }
$$

a complete set of $\pi$-commuting to each other conservation laws of the Kontsevich dynamical system (4.7), thus proving its generalized integrability. Moreover, choosing both another group algebra and orbit elements $\tilde{1} \in \tilde{\mathcal{G}}^{*}$, one can construct the same way many other integrable Hamiltonian systems on the associative noncommutative phase space $A$, that is planned to be a topic of a next investigation.

\section{Conclusion}

In this work we succeeded in revisting the classical Poisson manifolds approach to Hamiltonian operators on functional noncommutative manifolds, as well as presented it simple and natural realization, generated by associative noncommutative group algebra. The latter appeared to be very useful for describing a wide class of new Lax type integrable nonlinear Hamiltonian systems on associative noncommutative algebras, interesting for diverse applications in modern quantum physics.

\section{Acknowledgements}

The authors are cordially indebted to Prof. Anatol Odziewicz for fruitful and instructive discussions during the XXXVIII Workshop on Geometric Methods in Physics held on Juny 30 - July 06, 2019 in Bialowieza, Poland. A.P. thanks also the Departments of Mathematical Sciences of the Rutgers University and the NJIT University of Technology (NJ, USA) for invitations to visit them during the Spring Semester-2019, where there was prepared an essential part of the present work. The aknowledgements belong to the Department of Physics, Mathematics and Computer Science of the Cracov University of Technology for a local research grant F-2/370/2018/DS.

\section{References}

1. Bogolyubov NN, Prikarpatskii AK (1990) A bilocal periodic problem for the Sturm-Liouville and Dirac operators and some applications to the theory of nonlinear dynamical systems. I. Ukrainian Math J 42: 702-707. Link: http://bit.ly/37vTqsJ

2. Drobotskaya IS (1993) The Poisson structures related with Lax integrable operator dynamical systems. Kiev 33.

3. Prykarpatsky AK, Bogolubov NN (1990) A Bilocal periodic problem for differential Sturm-Liouville and Dirac operators and some applications to the theory of nonlinear dynamical systems I. DAN SSSR Math 42: 702-707. (in Russian) Link: http://bit.ly/30X3Grm

4. Prykarpatsky A, Mykytyuk I (1998) Algebraic integrability of nonlinear dynamical systems on manifolds: classical and quantum aspects. Kluwer Academic Publishers, the Netherlands

5. Kontsevich M (1993) Formal (non)-commutative symplectic geometry. In Israe M. Gelfand, Lawrence Corwin, and James Lepowsky, editors, The Gelfand Mathematical Seminars, 1990-1992. 173-187. Link: http://bit.ly/201iMHe

6. Kontsevich M (2011) Noncommutative Identities. Link: http://bit.ly/203n802

7. Kontsevich M, Rosenberg A (2000) Noncommutative smooth spaces In The Gelfand mathematical seminars, 1996-1999. 85-108. Link: http://bit.ly/30YX0ZG

8. Mikhailov AV, Sokolov VV (2000) Integrable ODEs on associative algebras. Communications in Mathematical Physics 211: 231-251. Link: http://bit.ly/2uAR3Gm

9. Odesskii AV, Rubtsov VN, Sokolov VV (2012) Bi-Hamiltonian ordinary differential equations with matrix variables. Theoretical and Mathematical Physics 171: 442-447. Link: http://bit.ly/30XMGRP

10. Odesskii A, Rubtsov V, Sokolov V (2013) Poisson brackets on free associative algebras. Conteprorary Mathematics 592: 295. 
11. Olver PJ, Sokolov VV (1998) Integrable evolution equations on associative algebras. Communications in Mathematical Physics 193: 245-268. Link: http://bit.ly/3aLymAA

12. Abraham R, Marsden JE (1978) Foundations of mechanics. Benjamin/ Cummings Publisher.

13. Arnold VI (1989) Mathematical methods of classical mechanics. Link: http://bit.ly/2kyqyNm

14. Blaszak M (1998) Bi-Hamiltonian dynamical systems. NY, Springer.

15. Blackmore D, Prykarpatsky AK, Samoylenko VH (2011) Nonlinear dynamica systems of mathematical physics. World Scientific Publisher, NJ, USA 564. Link: http://bit.ly/38JfkJe

16. Oevel W (1988) Dirac constraints in field theory: Lifts of Hamiltonian systems to the cotangent bundle. J Math Phys 29: 210-219. Link: http://bit.ly/2U2dGya

17. Olver PJ (1993) Applications of Lie Groups to Differential Equations. Second Edition, Springer-Verlag, New York. Link: http://bit.ly/2RGq4Cr

18. Gel'fand IM, Dorfman IYA (1979) Hamiltonian operators and algebraic structures related to them. Funct Anal Appl 13: 248-262. Link: http://bit.ly/31itiz

19. Lichnerovich A (1977) Les varietes de Poisson at leurs algebres de la associees. J Diff Geometry 12: 253-300. Link: http://bit.ly/38A4SDM

20. Sergyeyev A (2004) A simple way of making a Hamiltonian systems into a bi-Hamiltonian one. Acta Applicandae Mathematica 83 : 183-197. Link: http://bit.ly/36vK6n6

21. Mokhov OI (2004) Symplectic and Poisson geometry on loop spaces of smooth manifolds and integrable equations. Computer Science Institute Publisher, Moscow.

22. Balinski AA, Novikov SP (1985) Poisson brackets of hydrodynamic type Frobenius algebras and Lie algebras. 32: 228-231. Link: http://bit.ly/37vSJ2z

23. Ayupov S, Omirov B (1998) On Leibniz algebras. In: Algebra and Operato Theory (Tashkent, 1997), Kluwer Acad. Publ Dordrecht, 1-12. Link: http://bit.ly/2GtBcfn

24. Artemovych OD, Balinsky AA, Blackmore D, Prykarpatski AK (2018) Reduced Pre-Lie Algebraic Structures, the Weak and Weakly Deformed BalinskyNovikov Type Symmetry Algebras and Related Hamiltonian Operators. Symmetry 10: 601. Link: http://bit.ly/36z5MPf

25. Blackmore D, Prykarpatsky YA, Bogolubov NN Prykarpatski AK (2014) Integrability of and differential-algebraic structures for spatially 1D hydrodynamical systems of Riemann type. Chaos Solitons Fractals 59: 59-81. Link: http://bit.ly/2vrSo2P

26. Prykarpatsky YA, Artemovych OD, Pavlov M, Prykarpatsky AK (2012) The differential-algebraic and bi-Hamiltonian integrability analysis of the Riemann type hierarchy revisited. J Math Phys 53: 103521. Link: http://bit.ly/2vlpvVL

27. Prykarpatsky YA, Artemovych OD, Pavlov M, Prykarpatsky AK (2013) The differential-algebraic and bi-Hamiltoinian integrability analyss of the Riemann type hydrodynamic systems. Rep Math Phys 71: 305-351. Link: http://bit.ly/38Jt8U8

28. Arthamonov S (2015) Noncommutative inverse scattering method for the Kontsevich system. Lett Math Phys 105: 1223-1251. Link: http://bit.ly/38LM8kT

29. Arthamonov S (2017) Modi ed double Poisson brackets. J Algebra 492: 212 233. Link: http://bit.ly/37vmcd3
30. Van den Bergh M (2008) Double Poisson algebras. Trans Amer Math Soc 360 5711-5769. Link: http://bit.ly/200lo86

31. Efimovskaya O, Wolf T (2012) On Integrability of the Kontsevich Non-Abelian ODE system. Lett Math Phys 100: 161-170. Link: http://bit.ly/2GtUNw9

32. Faddeev LD, Takhtadjan LA (1986) Hamiltonian methods in the theory of solitons. Springer, New York, Berlin.

33. Reyman AG, Semenov-Tian-Shansky MA (1983) Integrable Systems, The Computer Research Institute Publ., Moscow-Izhvek, 2003 (in Russian) Semenov-Tian-Shansky, M. What is a classical R-matrix? Func Anal Appl 17: 259-272.

34. Lax PD (1968) Integrals of nonlinear equations of evolution and solitary waves Comm Pure Applied Math 21: 467-490. Link: http://bit.ly/2RwIntu

35. Novikov S, Manakov SV, Pitaevskii LP, Zakharov VE (1984) Theory of Solitons The Inverse Scattering Method. Monographs in Contemporary Mathematics, Springer. Link: http://bit.ly/2Zov536

36. Golenia J, Pavlov MV, Popowicz Z, Prykarpatsky AK (2010) On a nonlocal Ostrovsky-Whitham type dynamical system, its Riemann type inhomogenious regularizations and their integrability. Sigma 6: 1-13. Link: http://bit.ly/30WYILe

37. Oevel W (1989) R-structures, Yang-Baxter equations and related involution theorems. Journ Math Phys 30: 1140-1149. Link: http://bit.ly/2RM00o4

38. Prykarpatsky AK, Artemovych OD, Popowicz Z, Pavlov MV (2010) Differentialalgebraic integrability analysis of the generalized Riemann type and Kortewegde Vries hydrodynamical equations. J Phys A Math Theor 43: 295205. Link: http://bit.ly/2RXbYeG

39. Prykarpatsky A, Prytula MM (2006) The gradient-holonomic integrability analysis of a Whitham-type nonlinear dynamical model for a relaxing medium with spatial memory. Nonlinearity 19: 2115-222. Link: http://bit.ly/3al9tFH

40. Semenov-Tian-Shansky MA (1983) What is a classical R-matrix? Func Anal Appl 17: 259-272. Link: http://bit.ly/37xaGOL

\section{Discover a bigger Impact and Visibility of your article publication with}

\section{Peertechz Publications}

\section{Highlights}

* Signatory publisher of ORCID

* Signatory Publisher of DORA (San Francisco Declaration on Research Assessment)

- Articles archived in worlds' renowned service providers such as Portico, CNKI, AGRIS, TDNet, Base (Bielefeld University Library), CrossRef, Scilit, J-Gate etc.

* Journals indexed in ICMJE, SHERPA/ROMEO, Google Scholar etc.

* OAI-PMH (Open Archives Initiative Protocol for Metadata Harvesting)

* Dedicated Editorial Board for every journal

* Accurate and rapid peer-review process

* Increased citations of published articles through promotions

* Reduced timeline for article publication

Submit your articles and experience a new surge in publication services

(https://www.peertechz.com/submission).

Peertechz journals wishes everlasting success in your every endeavours.

Copyright: ( 2020 Hentosh OE, et al. This is an open-access article distributed under the terms of the Creative Commons Attribution License, which permits unrestricted use, distribution, and reproduction in any medium, provided the original author and source are credited.

Citation: Hentosh OE, Balinsky AA, Prykarpatski AK (2020) Poisson structures on (non)associative noncommutative algebras and integrable Kontsevich type Hamiltonian systems. Ann Math Phys 3(1): 001-006. DOI: https://dx.doi.org/10.17352/amp.000010 